\title{
A NEW EVANESCENT-MODE FILTER FOR DENSELY PACKAGED WAVEGUIDE APPLICATIONS
}

\author{
Vladimir A. Labay and Jens Bornemann
}

\author{
Laboratory for Lightwave Electronics, Microwaves and Communications \\ (LLiMiC) \\ Department of Electrical and Computer Engineering \\ University of Victoria, Victoria, B.C. Canada
}

\begin{abstract}
A novel cvanescent-mode filter structure based on $\mathrm{T}$ septum waveguide technology is presented for applications in extremely compact designs. The excellent characteristics of the T-septum waveguide are utilized to significantly reduce the resonator cutoff frequency compared with standard ridge waveguide designs. This allows the evanescent-mode guide to become smaller in cross-section, hence considerably reducing the overall length of the filter. The computer-aided design uses a full-wave mode-matching technique which takes into account higher-order mode coupling at all discontinuities involved. Design data are given for $\mathrm{X}$ - and $\mathrm{Ka}$-band. The method is verified by measurements. The manufactured $10 \mathrm{GHz}$ prototype measures only $18.4 \mathrm{~mm}$ in length.
\end{abstract}

\section{INTRODUCTION}

Microwave filters in evanescent-mode waveguide technology are well known for their wide stopbands and their small size compared to conventional coupled resonator filters. The techniques commonly applied to create a resonator in the below-cutoff waveguide sections include capacitive screws [1, 2], round posts [3, 4], ridges [5-7] and E-plane fins [8].

The size of a complete evanescent-mode filter structure decreases with the cross-section of the waveguide operating below cutoff since, at the same time, the filter's coupling sections considerably decrease in length. This tendency, however, imposes higher requirements on the resonator structure because its cross-section must provide for a reduction in the cutoff frequency of the below-cutoff guide to approximately that of the much larger input/output waveguide. In this respect, the designs offered so far [1-8] show only moderate success.

Therefore, this paper focuses on a new type of evanescent-mode waveguide filter (Fig. 1) utilizing T-septum technology [9]. Since the T-septum offers increased performance in terms of bandwidth enhancement and cutoff frequency reduction $[10,11]$ compared with the approaches mentioned above, this technology is ideally suited for evanescent-mode filter applications. The T-septum filter design is carried out using mode-matching techniques, e.g. [6], which include higher-order mode interactions at all discontinuities involved, and standard optimization procedures. Design examples are given for X-and Ka-band applications, and the theory is verified by measured results. The advantage of this new evanescent-mode filter in terms of size reduction is demonstrated by the realized $10 \mathrm{GHz}$ prototype which measures only $18.4 \mathrm{~mm}$ in length.

\section{THEORY}

For the computer-aided analysis and design of the $T$ septum evanescent-mode filter, the mode-matching technique $[6,12]$ is applied. The resulting generalized scattering matrix includes the discontinuity effects and higher-order mode interactions throughout the component.

The electromagnetic field in each longitudinal filter section (c.f. Fig. 2a)

$$
\begin{aligned}
& \overrightarrow{\boldsymbol{E}}=\frac{1}{j \omega \varepsilon} \nabla \times \nabla \times\left(A_{e z} \overrightarrow{\boldsymbol{e}}_{z}\right)+\nabla \times\left(A_{h z} \overrightarrow{\boldsymbol{e}}_{z}\right) \\
& \overrightarrow{\boldsymbol{H}}=\frac{-1}{j \omega \mu} \nabla \times \nabla \times\left(A_{h z} \vec{e}_{z}\right)+\nabla \times\left(A_{e z} \vec{e}_{z}\right)
\end{aligned}
$$

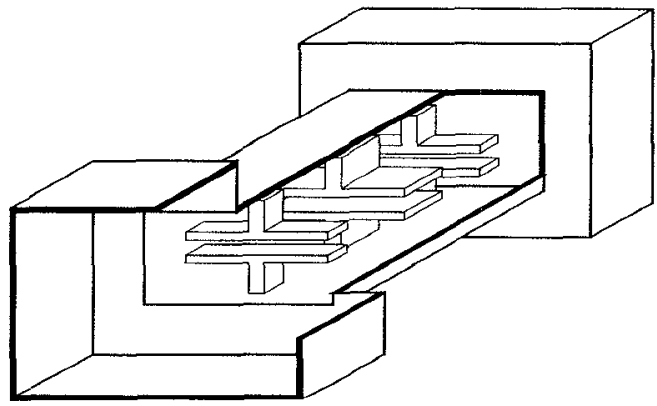

Fig. 1 Evanescent-mode T-septum waveguide filter. 
is derived from vector potential components

$$
\begin{aligned}
& A_{h z}=\sum_{m=0}^{M} \sum_{n=0}^{N} \sqrt{Z_{h m n}} T_{h m n}(x, y)\left(F_{h m n} e^{-j k_{2 h m n^{2}}}+B_{h m n}^{v} e^{+j k_{2 h m n}{ }^{2}}\right) \\
& A_{e z}=\sum_{m=1}^{M} \sum_{n=1}^{N} \sqrt{Y_{e m n}} T_{e m n}(x, y)\left(F_{e m n} e^{-j k_{z e m n}{ }^{2}}-B_{e m n} e^{+j k_{z e m n^{2}}}\right)
\end{aligned}
$$

which are specified in terms of wave impedances/admittances, cross-section functions, and forward and backward traveling waves as given in [12]. For each combination $m n$ in (3), (4), the cross-section function of the T-septum waveguide is obtained by superposition of subregions I to III (Fig. 2b).

$$
\begin{aligned}
T_{h}(x, y)= & \sum_{p=0}^{p-1} A_{p}^{I} \frac{\sin \left\{k_{x p}^{I}\left(x-\frac{a}{2}\right)\right\}}{k_{x p}^{I}} \frac{\cos \left\{\frac{p \pi}{b_{1}} y\right\}}{\sqrt{1+\delta_{o p}}} \\
& +\sum_{q=0}^{Q-1} A_{q}^{I I} \cos \left\{k_{x q}^{I I} x\right\} \frac{\cos \left\{\frac{2 q \pi}{b} y\right\}}{\sqrt{1+\delta_{o q}}} \\
& +\sum_{r=0}^{R-1} A_{r}^{I I I} \cos \left\{k_{x r}^{I I I}\left(x-a_{2}\right)\right\} \frac{\cos \left\{\frac{r \pi}{b-b_{2}}\left(y-b_{2}\right)\right\}}{\sqrt{1+\delta_{o r}}}
\end{aligned}
$$

$\mathbf{a}$
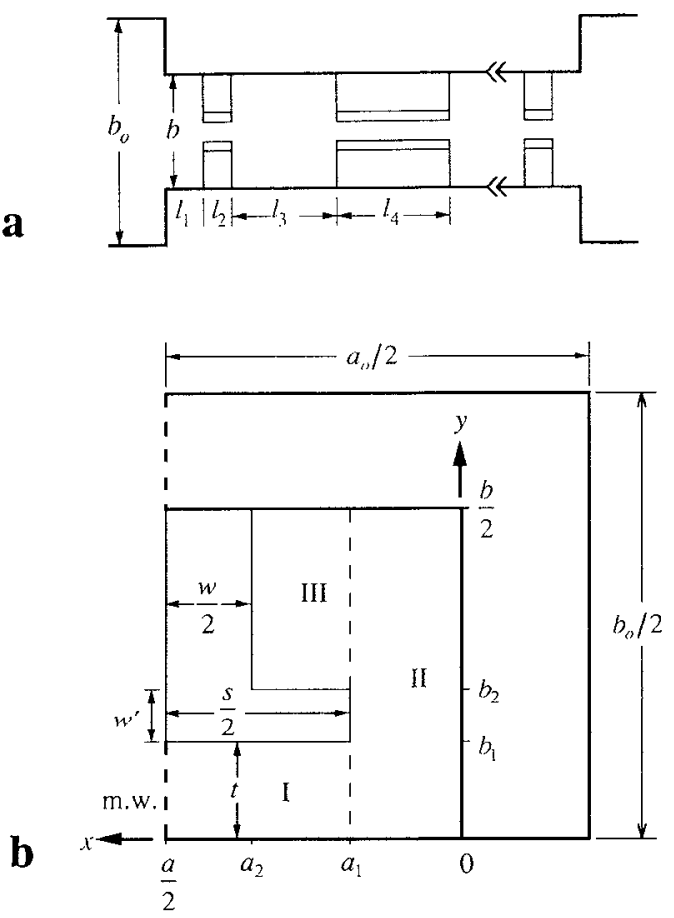

Fig. 2 Longitudinal section dimensions (a) and cross-sectional dimensions (b; only one quarter shown) of evanescentmode T-septum waveguide filter.

$$
\begin{aligned}
T_{e}(x, y)= & \sum_{p=1}^{P-1} D_{p}^{I} \cos \left\{k_{x p}^{I}\left(x-\frac{a}{2}\right)\right\} \sin \left\{\frac{p \pi}{b_{1}} y\right\} \\
& +\sum_{q=1}^{Q-1} D_{q}^{I I} \frac{\sin \left\{k_{x q}^{I I} x\right\}}{k_{x q}^{I I}} \sin \left\{\frac{2 q \pi}{b} y\right\} \\
& +\sum_{r=1}^{R-1} D_{r}^{I I I} \frac{\sin \left\{k_{x r}^{I I}\left(x-a_{2}\right)\right\}}{k_{x r}^{I I I}} \sin \left\{\frac{r \pi}{b-b_{2}}\left(y-b_{2}\right)\right\}
\end{aligned}
$$

The separation constants $k_{x}$ and amplitude coefficients $A, D$ in (5), (6) are determined by the solution of the characteristic matrix equation [13] and subsequent power normalization [12], respectively.

Sufficient convergence behavior can be observed if the design is carried out considering $35 \mathrm{TE}$ and $\mathrm{TM}$ modes and $\mathrm{Q}=$ 10 expansion terms for the determination of the T-septum eigenfunctions. For given $Q$, the number of terms in subregions I and III (Fig. 2b) are specified by the y-direction dimensions ratios. With this combination of modes, the analysis of one set of filter parameters at 50 frequency samples requires a CPU time of about 10 minutes on an IBM RS6000/530 workstation.

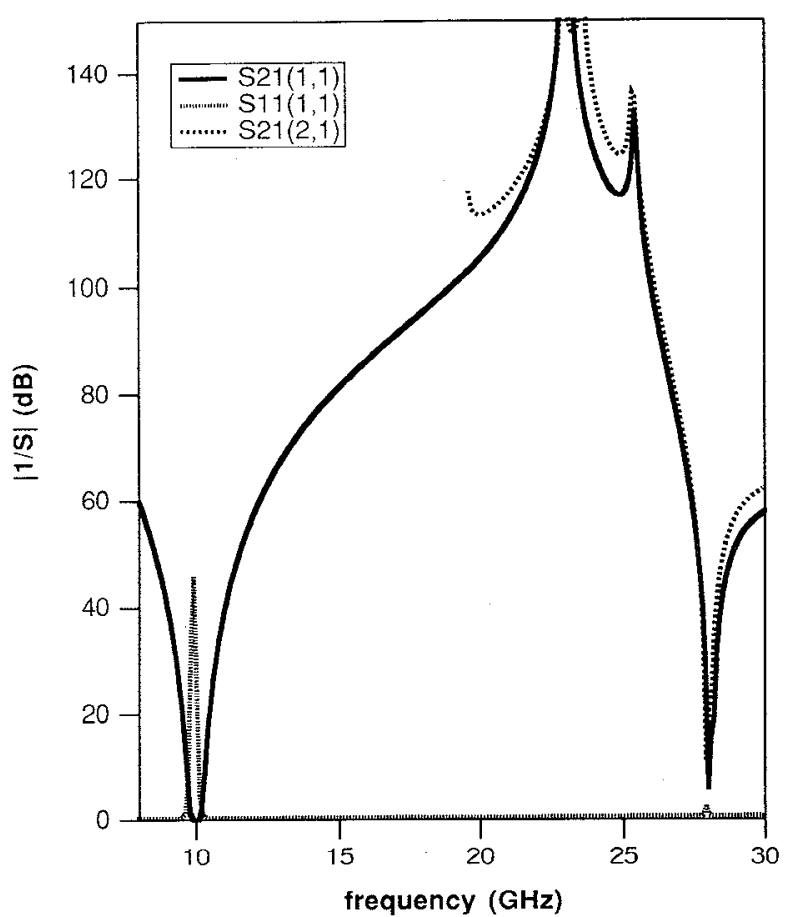

Fig. $3 \mathrm{X}$-band three-resonator evanescent-mode $\mathrm{T}$-septum waveguide filter. Dimensions (c.f. Fig. 2): $\mathrm{a}_{0}=22.85 \mathrm{~mm}, \mathrm{~b}_{0}=10.16 \mathrm{~mm}, \mathrm{a}=7.06 \mathrm{~mm}, \mathrm{~b}=6.98 \mathrm{~mm}$, $\mathrm{w}=2 \mathrm{~mm}, \quad \mathrm{w}^{\prime}=1 \mathrm{~mm}, \mathrm{~s}=5.06 \mathrm{~mm}, \quad \mathrm{~b}_{1}=0.49 \mathrm{~mm}$, $\mathrm{l}_{1}=\mathrm{l}_{7}=0.5 \mathrm{~mm}, \mathrm{l}_{2}=\mathrm{l}_{6}=0.49 \mathrm{~mm}, \mathrm{l}_{3}=\mathrm{l}_{5}=7.75 \mathrm{~mm}$, $1_{4}=0.94 \mathrm{~mm}$. 


\section{RESULTS}

Fig. 3 shows the response of a three-resonator evanescent-mode filter for $10 \mathrm{GHz}$ in $\mathrm{X}$-band. The design is optimized for a bandwith of $400 \mathrm{MHz}$ and a return loss better that $35 \mathrm{~dB}$. Although the cutoff frequency of the reduced-size waveguide is $21 \mathrm{GHz}$, the second passband is shifted beyond $27.5 \mathrm{GHz}$. If a symmetric configuration is assumed, then the next higher-order mode in the $\mathrm{X}$-band output waveguide begins to propagate at $19.7 \mathrm{GHz}$. As the dashed line shows, this fact does not contribute to any deterioration of the filter response at higher frequencies. Note that at the second harmonic frequency at $30 \mathrm{GHz}$, attenuation values greater than $55 \mathrm{~dB}$ are obtained which makes the design suitable for $\mathrm{X}$-band communication systems applications.

Fig. 4 shows a five-resonator structure for comparison with Fig. 3. Note that not only the skirt selectivity and stopband attenuation is significantly improved as it could be expected by adding two resonators, but also that the attenuation at the second harmonic $(30 \mathrm{GHz})$ almost doubled.

A three-resonator millimeter-wave design for $33 \mathrm{GHz}$ in Ka-band and standard W-band below-cutoff section is presented in Fig. 5. The calculated minimum passband return loss is $38 \mathrm{~dB}$. The optimized filter component has a length of only $7.4 \mathrm{~mm}$ and excellent stopband characteristics up to 73 $\mathrm{GHz}$.

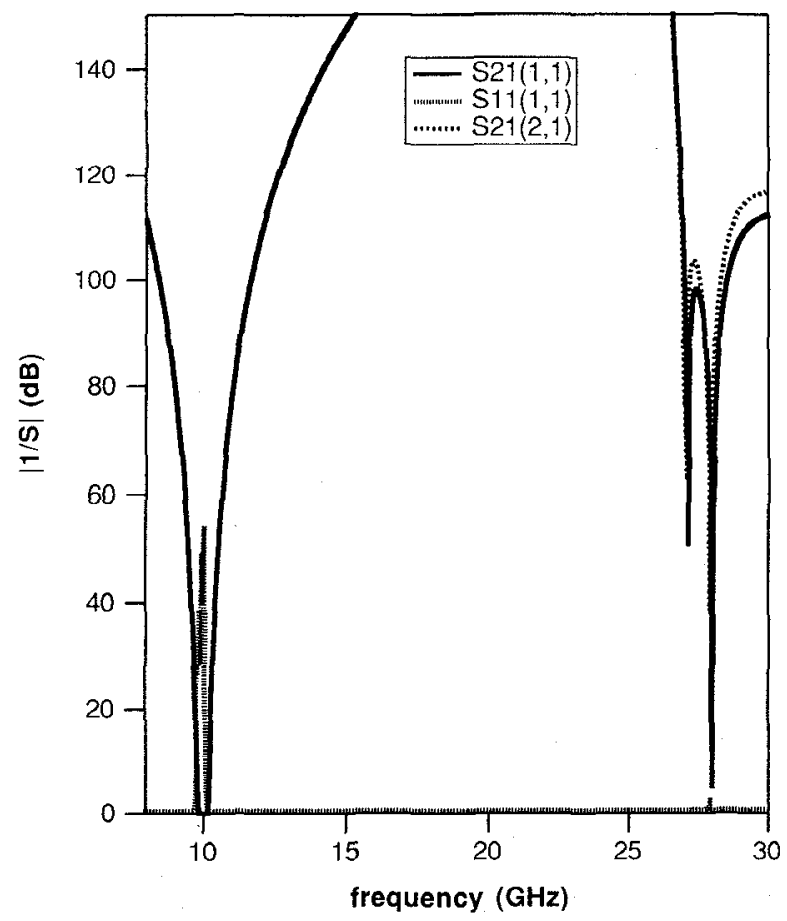

Fig. 4 X-band five-resonator evanescent-mode T-septum waveguide filter. Dimensions: $l_{1}=l_{11}=0.47 \mathrm{~mm}$ $1_{2}=1_{10}=0.508 \mathrm{~mm}, l_{3}=19=7.82 \mathrm{~mm}, l_{4}=16=18=0.981 \mathrm{~mm}$, $1_{5}=17=8.54 \mathrm{~mm}$; other dimensions as in Fig. 3 .
Fig. 6 shows a comparison between theoretical results and those obtained by measurements. Due to severe limitations in the manufacturing process, the solid-brass prototype of only $18.4 \mathrm{~mm}$ length could not be realized with the desired acuracy of $+/-10 \mu \mathrm{m}$. With the actually realized dimensions as input parameters, however, good agreement with the measured data is obtained. The differences in passpand return and insertion loss are due to fact that the theory assumes a symmetric connection to the $\mathrm{X}$-band waveguides whereas the prototype shows slight offsets in both $\mathrm{x}$ and $\mathrm{y}$ directions. This transition seems to be the critical point in the design. However, it is believed that the filter structure will perform as optimized when numerically controlled milling facilities are used for the manufacturing process of the complete component.

\section{CONCLUSIONS}

A new compact evanescent-mode filter design in $\mathrm{T}$ septum waveguide technology is presented. Since the resonator cutoff frequency is significantly reduced by inserting a $T$ septum instead of the commonly used ridge in the below-cutoff waveguide, the cross-section of this guide can be reduced, hence considerably reducing the lengths of the coupling sections and, therefore, of the overall structure. The results of the computer-aided design, which is carried out using a fullwave mode-matching technique taking into account higherorder mode coupling at all discontinuities, agree well with measurements as is demonstrated for an $10 \mathrm{GHz} \mathrm{X}$-band prototype of only $18.4 \mathrm{~mm}$ length. Other design data are given for X- and Ka-band.

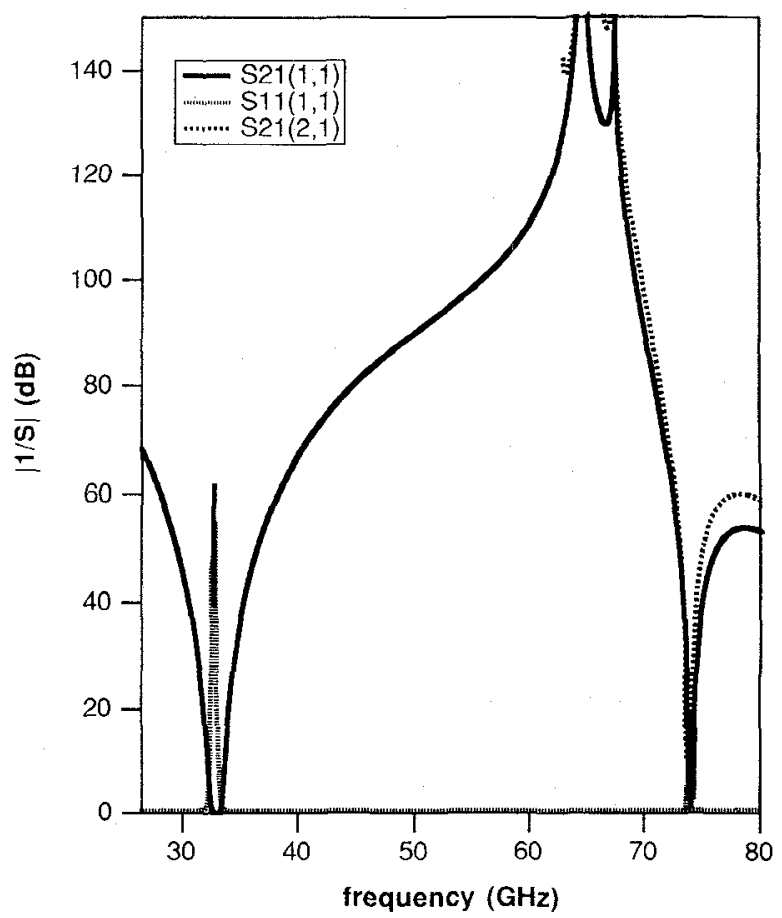

Fig. 5 Millimeter-wave three-resonator evanescent-mode Tseptum filter. Dimensions: $\mathrm{a}_{0}=2 \mathrm{~b}_{0}=7.112 \mathrm{~mm}(\mathrm{Ka}-$ band), $a=2 b=2.54 \mathrm{~mm}$ (W-band), $w=w^{\prime}=0.5 \mathrm{~mm}$, $\mathrm{s}=2.04 \mathrm{~mm}, \quad b_{1}=0.1 \mathrm{~mm}, l_{1}=17=0.2 \mathrm{~m} \mathrm{~m}$, $\mathrm{l}_{2}=\mathrm{l}_{6}=0.159 \mathrm{~mm}, \mathrm{l}_{3}=\mathrm{l}_{5}=3.187 \mathrm{~mm}, \mathrm{l}_{4}=0.297 \mathrm{~mm}$. 


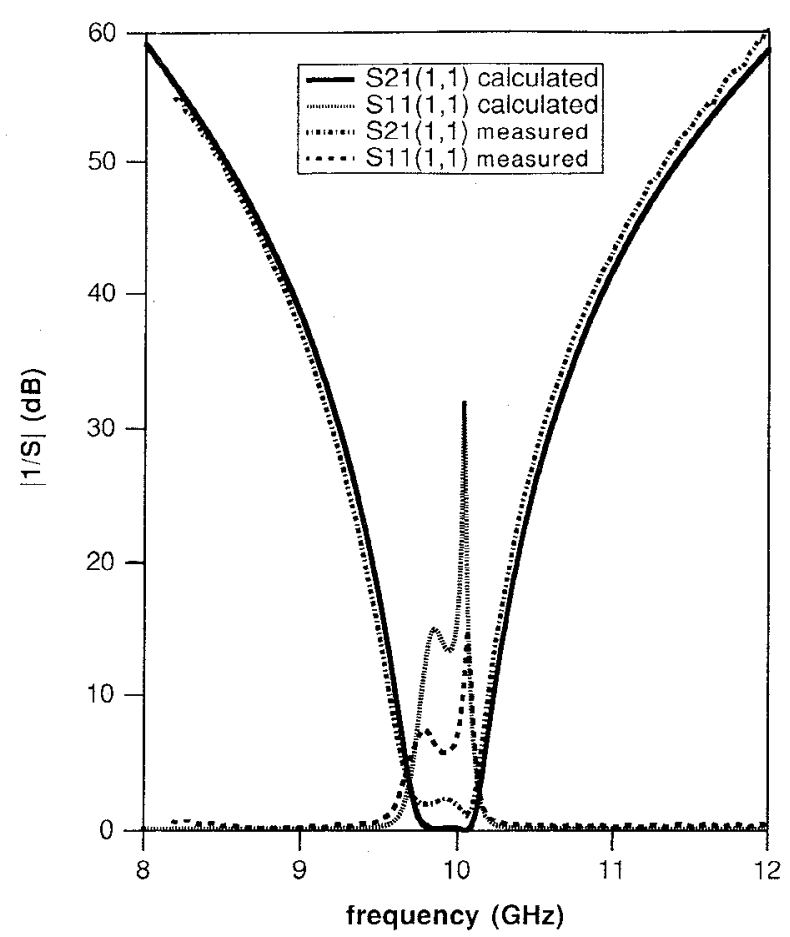

Fig. 6 Comparison between measured and calculated responses of an X-band three-resonator T-septum waveguide prototype.

\section{REFERENCES}

[1] G. Craven, "Waveguide band-pass filters using evanescent modes", Electron. Lett., vol. 2, pp. 251-252, July 1966.

[2] G. Craven and C.K. Mok, "The design of evanescent mode waveguide band-pass filters for a prescribed insertion loss characteristic", IEEE Trans. Microwave Theory Tech., vol. MTT-19, pp. 295-308, Mar. 1971.

[3] H.F. Chappell, "Waveguide low pass filter using evanescent mode inductors, " Microwave J., vol. 21, pp. 71-72, Dec. 1978.

[4] R. V. Snyder, "Broadband waveguide or coaxial filters with wide stopbands, using a stepped-wall evanescent mode approach", Microwave J., vol. 26, pp.83-88, Dec. 1983.

[5] A.M.K. Saad, "Novel lowpass harmonic filters for satellite application", in 1984 IEEE MTT-S Intl. Microwave Symp. Dig., pp. 292-294.

[6] J. Bornemann and F. Arndt, "Transverse resonance, standing wave, and resonator formulations of the ridge waveguide eigenvalue problem and its application to $\mathrm{E}$ plane finned waveguide filters", IEEE Trans. Microwave Theory Tech., vol. 38, pp. 1104-1113, Aug. 1990.

[7] J.-W. Tao and H. Baudrand, "Multimodal variational analysis of uniaxial waveguide discontinuities", IEEE Trans. Microwave Theory Tech., vol. 39, pp. 506-516, Mar. 1991.

[8] Q. Zhang and T. Itoh, "Computer-aided design of evanescent-mode waveguide filters with non-touching E-plane fins", IEEE Trans. Microwave Theory Tech., vol. MTT-36, pp. 404-412, Feb. 1988.
[9] R.S. Elliott, "Two-mode waveguide for equal mode velocities", IEEE Trans. Microwave Theory Tech., vol. MTT-16, pp. 282-286, May 1968.

[10] Y. Zhang and W.T. Joines, "Some properties of Tseptum waveguides", IEEE Trans. Microwave Theory Tech., vol. MTT-35, pp. 769-775, Aug. 1987.

[11] F.G. German and L.S. Riggs, "Bandwidth properties of rectangular T-septum waveguides", IEEE Trans. Microwave Theory Tech., vol. 37, pp. 917-919, May 1989.

[12] J. Bornemann, "Comparison between different formulations of the transverse resonance field-matching technique for the three-dimensional analysis of metalfinned waveguide resonators", Intl. J. Numerical Modelling, vol. 4, pp. 63-73, Mar. 1991.

[13] V.A. Labay and J. Bornemann, "Matrix singular value decomposition for pole-free solutions of homogeneous matrix equations as applied to numerical modelling methods", IEEE Microwave Guided Wave Lett., vol. 2, Feb. 1992. 